\title{
EFEITO DO GLICEROL NAS PROPRIEDADES MECÂNICAS DE FILMES A BASE DE QUITOSANA
}

Effect of glycerol on the mechanical properties of films the based on chitosan

Efecto del glicerol sobre las propiedades mecánicas de películas a base de quitosana

Laiza Bernardes Antunes ${ }^{1}$, Sabrina Silva Gonçalves ${ }^{1}$, Miriam Fontes Araujo Silveira $^{2}$, Adriana Régia Marques de Souza ${ }^{2}$, Deivis de Moraes Carvalho ${ }^{3}$

${ }^{1}$ Discente do curso de Engenharia de Alimentos da Universidade Federal de Goiás, Goiânia-GO, Brasil

${ }^{2}$ Docente da Escola de Agronomia da Universidade Federal de Goiás, Goiânia-GO, Brasil

${ }^{3}$ Mestre em Ciência e Tecnologia de Alimentos, Técnico da Escola de Agronomia da Universidade Federal de Goiás, Goiânia-GO, Brasil

*Correspondência: Escola de Agronomia, Rodovia Goiânia-Nova Veneza, Km 0, s/n - Campus Samambaia, Goiânia, Goiás, Brasil. CEP: 74690900.e-mail sabrinasilvagoncalves615@gmail.com

\section{RESUMO}

A quitosana é um polímero com excelente capacidade de formação de gel. Possui ação antimicrobiana e é um material completamente degradado por microrganismos em processo de decomposição. O objetivo deste trabalho foi desenvolver filmes biodegradáveis a base de quitosana e avaliar o efeito da adição de glicerol nas suas propriedades mecânicas. Os filmes foram obtidos pelo método casting a partir de soluções filmogênicas com $2 \%$ de quitosana, $5 \%$ de ácido acético glacial e diferentes concentrações de glicerol $(0,5 ; 0,75$ e 1,0\%). Os filmes foram caracterizados quanto à espessura, propriedades de tração e teste de perfuração. Apresentaram espessura média de 46,0 $\pm 5,0 \mu \mathrm{m}$, coloração amarelada, homogêneos e com boa maleabilidade. O filme com $0,5 \%$ de glicerol suportou uma tensão significativamente maior que os demais. A adição de $0,75 \%$ e 1,0\% de glicerol causou uma redução de 34,4 \% e 39,9 $\%$ nesta propriedade, respectivamente, em relação ao filme com $0,5 \%$ de glicerol. Quanto ao módulo de elasticidade, o aumento da concentração do plastificante causou redução da rigidez dos filmes na região de comportamento elástico. As diferentes concentrações utilizadas de glicerol não interferiram significativamente nos parâmetros de elongação e teste de perfuração.

Palavras-chave: plastificante, polímero, quitina.

\begin{abstract}
Chitosan is a polymer with excellent gel formation ability. It has an antimicrobial action and is a material completely degraded by microorganisms in the process of decomposition. The objective of this work was to develop biodegradable films based on chitosan and to evaluate the effect of the addition of glycerol on its mechanical properties. The films were obtained by the method casting starting from solutions filmogênicas with $2 \%$ of quitosana, $5 \%$ of glacial acetic acid and different glycerol concentrations $(0,5 ; 0,75$ and 1,0\%). The films were characterized as for the thickness, traction properties and perforation test. They presented medium thickness of 46,0 $\pm 5,0 \mu \mathrm{m}$, coloration yellowish, homogeneous, with good malleableness. The film with 0,5\% of glycerol supported a significantly higher stress than the others. The addition of $0.75 \%$ and $1.0 \%$ glycerol caused a $34.4 \%$ and $39.9 \%$ reduction in this property, respectively, relative to the film with $0.5 \%$ glycerol. As for the modulus of elasticity, the increase of the plasticizer concentration caused a reduction of the stiffness of the films in the region of elastic behavior. The different concentrations of glycerol used did not have a significantly interfere in the elongation and drilling test parameters.
\end{abstract}

Keywords: plasticizer, polymer, chitin.

\section{RESUMEN}

La quitosana es un polímero con excelente capacidad de formación de gel. Posee acción antimicrobiana y es un material completamente degradado por microorganismos en proceso de descomposición. El objetivo de este trabajo fue desarrollar películas biodegradables a base de quitosana y evaluar el efecto de la adición de glicerol en sus 
propiedades mecánicas. Las películas fueron obtenidas por el método casting a partir de soluciones filmogénicas con $2 \%$ de quitosana, $5 \%$ de ácido acético glacial y diferentes concentraciones de glicerol (0,5, 0,75 y 1,0\%). Las películas se caracterizó en el grosor, las propiedades de tracción y la prueba de perforación. Se presentaron espesor medio de 46,0 \pm 5,0 $\mu \mathrm{m}$, coloración amarillenta, homogéneos y con buena maleabilidad. La película con 0,5\% de glicerol soportó una tensión significativamente mayor que los demás. La adición de 0,75\% y 1,0\% de glicerol causó una reducción del 34,4\% y del 39,9\% en esta propiedad, respectivamente, en relación con la película con un 0,5\% de glicerol. En cuanto al módulo de elasticidad, el aumento de la concentración del plastificante causó reducción de la rigidez de las películas en la región de comportamiento elástico. La concentración de glicerol no afectó significativamente los parámetros de elongación y la prueba de perforación.

Descriptores: plastificante, polímero, quitina.

\section{INTRODUÇÃO}

Os exoesqueletos dos crustáceos são, na maioria das vezes, descartados por não serem totalmente comestíveis. Esse resíduo é rico em quitina, onde pode-se extrair a quitosana, polímero que possui ação antimicrobiana, beneficiando não somente a área alimentícia, como também a de fármacos. Sua utilização é uma alternativa para diminuição do impacto ambiental, causado com o crescente aumento da aquicultura.

A quitosana possui excelente capacidade de formação de gel para produção de filmes. Assim, nos últimos anos vem sendo muito estudada, pois apresenta grande variedade de aplicações devido à sua biocompatibilidade e biodegradabilidade. É derivada de biomassa abundante e de baixo custo (SZYMANSKA e WINNICKA, 2015).

Os biofilmes a base de quitosana é um material completamente degradado por microrganismos em processo de decomposição, o que é mais um atrativo para as indústrias, pois ajuda a reduzir o problema da poluição (ASSIS et al., 2009).

A solubilidade da quitosana se dá apenas em faixas de $\mathrm{pH}$ próximas ao seu $\mathrm{pKa}(\sim 6,3)$, formando um polímero catiônico pela protonação dos grupos amino que, ao ser vertido sobre uma superfície apolar, resulta em filmes de quitosana após a evaporação do solvente. Esses filmes apresentam razoáveis propriedades mecânicas, sendo avaliados como potenciais materiais para revestir frutos e hortaliças, formar cartilagens e tecidos artificiais e como membranas de separação e complexação de íns de metais tóxicos em meio aquoso (ASSIS, 2010).

O glicerol é uma matéria prima renovável que, por ser plastificante, ao ser introduzido em filmes biodegradáveis promove alterações significativas de suas propriedades e, assim, garante uma ampla faixa de aplicação. Sem a ação deste plastificante o filme tem uma estrutura rígida e quebradiça, o que se torna inviável a sua produção. Assim, verificou-se a grande importância da adição do glicerol ao filme no quesito maleabilidade.

Portanto, o presente trabalho teve como objetivo desenvolver filmes biodegradáveis a base de quitosana e avaliar o efeito da adição de glicerol nas suas propriedades mecânicas.

\section{MATERIAIS E MÉTODOS}

$\mathrm{O}$ experimento foi realizado no Setor de Engenharia de Alimentos da Escola de Agronomia da Universidade Federal de Goiás, Goiânia-GO, no Laboratório de Análises Físico-Químicas e no Laboratório Multiusuário de Análises de Textura, Reologia e HPLC (LabMulti). Para produção dos filmes biodegradáveis foi utilizada quitosana em pó, peso molecular médio e grau de desacetilação de $100 \%$, adquirida da empresa Polymar, em Fortaleza-CE. O glicerol (Glicerina bidestilada da Dinâmica Química Contemporânea, com PM 
$92,10)$ foi usado como plastificante.

\section{Preparo das soluções filmogênicas}

As soluções filmogênicas foram preparadas com $2 \%$ de quitosana e $5 \%$ de ácido acético glacial $(60,05 \mathrm{~g} / \mathrm{mol})$. Foram adicionadas diferentes concentrações de glicerol $(0,5 ; 0,75$ e $1,0 \%)$ e o volume foi completado com água destilada. A solução ficou em constante agitação no homogeneizador tipo TURRAX (Marca Marconi/modelo MA-102), até completa dissolução.

\section{Produção dos filmes biodegradáveis}

Os filmes foram produzidos pelo método casting. Em placas de petri de poliestino $(140 \mathrm{~mm}$ de diâmetro) foram adicionados $40 \mathrm{~mL}$ da solução filmogênica. As placas foram colocadas em superfície plana, deixando secar por três dias, até a formação completa dos filmes. Estes foram retirados das placas de petri, identificados e condicionados em temperatura de $25 \pm 1{ }^{\circ} \mathrm{C}$ e umidade relativa de $50 \pm 5 \%$, por 48 horas antes das análises (ASTMD882-10, 2010).

\section{Análise Subjetiva}

Refere-se à aparência do filme avaliada por observações táteis e visuais. O filme deve apresentar uma superfície homogênea e contínua. Assim, foram excluídos das análises aqueles filmes defeituosos que apresentaram fissuras e bolhas após o processo de secagem, ou quaisquer outros sinais que pudessem prejudicar as análises.

\section{Espessura}

A espessura foi determinada com um micrômetro digital com resolução de $0,0001 \mathrm{~mm}$
(Qualitylabor, modelo MEP/Q, Perus), pela média aritmética de 10 medidas aleatórias para cada filme.

\section{Propriedades de tração}

As propriedades de tensão máxima (MPa), tensão na ruptura (MPa), elongação (\%) e módulo de elasticidade ou Young (MPa) dos filmes foram determinadas na Máquina Universal de Ensaios (Instron, 3367, Grove City, USA). Foram preparados 10 corpos de prova de cada filme, com $1 \mathrm{~cm}$ de largura e $10 \mathrm{~cm}$ de comprimento, obtidos de acordo com a norma ASTM-D882-10 (ASTM, 2010), com adaptações, e submetidos à tração. Os filmes foram fixados por duas garras corrugadas distantes $8,0 \mathrm{~cm}$ entre si, com uma delas se movimentando a uma velocidade de $12 \mathrm{~mm} / \mathrm{min}$.

\section{Teste de perfuração}

$\mathrm{O}$ teste de perfuração foi realizado no texturômetro (Stable Micro System - Surrey, TA.XT.plus Texture Analyser, Inglaterra). Os corpos de prova com $6,5 \mathrm{~cm}$ de diâmetro foram fixados em suporte circular. Uma sonda metálica de ponta esférica, com diâmetro de $0,5 \mathrm{~cm}$ a uma velocidade de $25,2 \mathrm{~mm} / \mathrm{min}$, fez a perfuração das amostras. Foi determinada a força de perfuração (N) e o deslocamento na perfuração (mm).

\section{Análise estatística}

$\mathrm{O}$ experimento foi realizado em delineamento inteiramente casualizados (DIC) com três repetições. Os dados foram submetidos à Análise de Variância (ANOVA) e as diferenças entre os tratamentos avaliados pelo teste de Tukey, a 5\% de significância, com auxílio do programa estatístico Sisvar (FERREIRA, 2008). 


\section{RESULTADOS E DISCUSSÃO}

\section{Análise subjetiva}

Os filmes biodegradáveis a base de quitosana incorporado com glicerol apresentaram-se amarelados, homogêneos, com boa maleabilidade e sem bolhas ou rachaduras na superfície (Figura 1).

Alguns filmes do tratamento $\mathrm{T} 1 \quad(0,5 \%$ glicerol) apresentaram regiões rugosas junto ao perímetro e com aspecto quebradiço, logo após a secagem. Também Silva et al. (2015) observaram essas características nos filmes produzidos com $2 \%$ e $3 \%$ de quitosana, utilizando $0,1 \%$ de glicerol $(\mathrm{m} / \mathrm{v})$.

Figura 1. Filmes biodegradáveis a base de quitosana com diferentes concentrações de glicerol (T1 - 0,5\%; T2 - 0,75\% e T3 - 1,0\%).

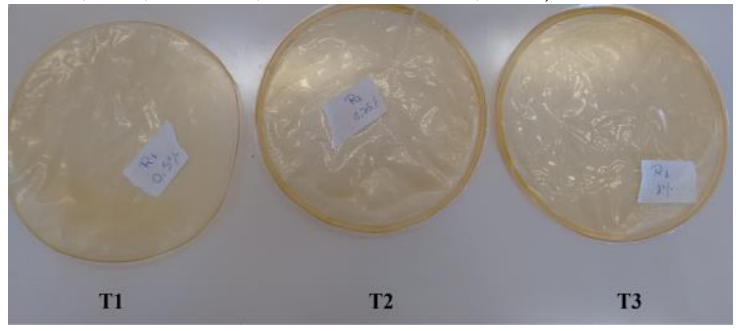

\section{Espessura}

Os filmes apresentaram espessura média de 46,0 $\pm 5,0 \mu \mathrm{m}$; não houve diferença significativa entre eles $(p>0,05)$. As propriedades mecânicas dos filmes podem sofrer influência da espessura. Segundo Rodríguez et al. (2012), o controle da espessura é muito importante, pois através dela pode-se avaliar a uniformidade dos polímeros, a repetibilidade da medida de suas propriedades e a validade das comparações entre os filmes.

De acordo com Mali et al. (2010), controlar a espessura dos filmes produzidos pelo método casting é uma etapa que exige bastante atenção. Para Carvalho (1997), Sobral (2000), Mali et al. (2004) e
Galdeano (2007), a espessura influencia largamente as propriedades mecânicas, principalmente a força na perfuração e a permeabilidade ao vapor de água de filmes hidrofílicos. Filmes mais espessos são mais resistentes à perfuração e menos permeáveis ao vapor de água.

Viégas (2016) produziu filmes biodegradáveis a partir de amido e quitosana, variando as proporções entre os dois polímeros. Verificou-se uma espessura de $120 \pm 10 \mu \mathrm{m}$ nos filmes com 2,0 \% (m/v) de quitosana e 1,0\% (v/v) de ácido acético. Percebe-se uma diferença considerável em relação à espessura dos filmes produzidos neste trabalho.

\section{Propriedades de tração}

Valores de tensão, módulo de elasticidade, elongação, entre outros, servem de base para comparação do desempenho mecânico dos polímeros. Portanto, a análise das propriedades mecânicas de materiais poliméricos é essencial para o processo de seleção do material usado no projeto de uma peça ou de um produto (SEBASTIÃO, 2003).

$\mathrm{Na}$ Tabela 1 estão apresentados os resultados obtidos das propriedades de tração dos filmes.

Segundo Garcia et al. (2000), a tensão de ruptura é a última tensão suportada pelo material antes da quebra. É definida como a força máxima necessária para que o filme deforme. Observa-se, pelos resultados obtidos, que o valor desta propriedade foi inversamente proporcional às concentrações de plastificante utilizadas.

O filme com $0,5 \%$ de glicerol suportou uma tensão significativamente maior que os 
demais filmes. A adição de $0,75 \%$ e $1,0 \%$ de glicerol causou uma redução de $34,4 \%$ e $39,9 \%$ nesta propriedade, respectivamente, em relação ao filme com menor concentração de glicerol $(0,5 \%)$. Isso significa que estes filmes não suportaram a mesma tensão aplicada ao filme com $0,5 \%$ de glicerol antes de se romperem. Também Dias
(2008), trabalhando com filmes de amido e farinha de arroz, observou uma diminuição considerável nos valores de tensão de ruptura com o aumento da concentração de plastificante, tornando-os mais flexíveis.

Tabela 1. Propriedades de tração dos filmes biodegradáveis a base de quitosana com diferentes concentrações de glicerol.

\begin{tabular}{cccc}
\hline Glicerol & Tensão de ruptura & Elongação & Módulo de elasticidade \\
$(\mathbf{\%})$ & $(\mathbf{M P a})$ & $(\boldsymbol{\%})$ & $(\mathbf{M P a})$ \\
\hline 0,5 & $7,50 \pm 0,45^{\mathbf{a}}$ & $9,95 \pm 0,73^{\mathbf{a}}$ & $155,15 \pm 3,95^{\mathbf{a}}$ \\
0,75 & $4,92 \pm 1,03^{\mathbf{b}}$ & $12,64 \pm 1,19^{\mathbf{a}}$ & $75,44 \pm 17,80^{\mathbf{b}}$ \\
1,0 & $4,51 \pm 0,55^{\mathbf{b}}$ & $14,93 \pm 4,60^{\mathbf{a}}$ & $51,99 \pm 12,58^{\mathbf{b}}$ \\
\hline
\end{tabular}

Nas colunas, médias seguidas das mesmas letras não diferem estatisticamente entre si, pelo teste de Tukey $(\mathrm{p}>0,05)$.

A elongação é a deformação total, relacionada com a variação do comprimento inicial e final sofrida pelo material durante a análise.

Observa-se que não houve diferença significativa $(p>0,05)$ na propriedade de elongação entre os filmes analisados. Porém, percebe-se uma tendência de aumento no valor desta propriedade com o aumento da concentração de glicerol. Segundo Galdeano et al. (2009), o plastificante age entre as cadeias poliméricas, reduzindo interações, aumentando a mobilidade e, consequentemente, tornando os filmes mais extensíveis.

O módulo de elasticidade ou módulo de Young é a relação entre a resistência à tração e a porcentagem de alongamento; corresponde à porção linear de uma determinada curva tensãodeformação sofrida pelo filme que pode ser ainda reversível. Indica a rigidez do filme, ou seja, quanto maior o valor do módulo de Young mais rígido será o filme (OLIVEIRA, 1996).

Observa-se que o filme com menor concentração de glicerol $(0,5 \%)$ apresentou maior valor do módulo de Young, diferindo significativamente dos demais filmes. O aumento da concentração do plastificante causou redução da rigidez dos filmes na região de comportamento elástico. Houve uma redução de $51,4 \%$ e de $66,5 \%$ deste parâmetro nos filmes com $0,75 \%$ e $1,0 \%$ de glicerol, respectivamente, em relação ao filme com $0,5 \%$. Portanto, estes filmes apresentaram-se menos rígidos e mais flexíveis.

\section{Teste de perfuração}

Os resultados obtidos no teste de perfuração 
estão apresentados na Tabela 2.

Tabela 2. Teste de perfuração dos filmes biodegradáveis a base de quitosana com diferentes concentrações de glicerol.

\begin{tabular}{ccc}
\hline $\begin{array}{c}\text { Glicerol } \\
(\%)\end{array}$ & $\begin{array}{c}\text { Força de perfuração } \\
(\mathbf{N})\end{array}$ & $\begin{array}{c}\text { Deslocamento na perfuração } \\
(\mathbf{m m})\end{array}$ \\
\hline 0,5 & $6,17 \pm 0,84^{\mathbf{a}}$ & $2,87 \pm 0,13^{\mathbf{a}}$ \\
0,75 & $4,90 \pm 0,50^{\mathbf{a}}$ & $3,04 \pm 0,02$ a \\
1,0 & $5,23 \pm 1,15^{\mathbf{a}}$ & $3,28 \pm 0,22$ a \\
\hline $\begin{array}{l}\text { Nas colunas, médias seguidas das mesmas letras não diferem estatisticamente entre si, pelo teste de Tukey } \\
(\mathrm{p}>0,05) .\end{array}$
\end{tabular}

Não houve diferença significativa $(p>0,05)$ nos parâmetros força de perfuração e deslocamento na perfuração entre os filmes analisados. Porém, percebeu-se uma leve tendência na redução da força de perfuração com o aumento da concentração de glicerol.

Rocha et al. (2014), trabalhando com filmes a base de amido de mandioca, proteína de soja e glicerol, relataram comportamento semelhante. Também Mali et al. (2004) observaram uma redução de 8,02 para 6,03 N neste parâmetro com o aumento da concentração de glicerol de 1,3 para 2,0 $\%$, em filmes de amido de cará. Este comportamento se deve ao rompimento de interações intermoleculares ocasionado pela presença do plastificante (GALDEANO et al., 2009).

Estudos realizados com filmes biodegradáveis demonstram que um método eficaz de reforçar as propriedades mecânicas é a mistura de polímeros (DIAS, 2013). Li et al. (2010) desenvolveram blendas de amido e quitosana e obtiveram aumento significativo da resistência à tração destes filmes com o aumento da porcentagem de quitosana. Bourtoom e Chinnan (2008), trabalhando com blendas de filmes biodegradáveis de amido de arroz e quitosana, perceberam um aumento da resistência à ruptura com a adição de quitosana.

\section{CONCLUSÃO}

Os filmes com $0,5 \%$ de glicerol apresentaram-se mais resistentes e com menor deformação na ruptura. Os filmes com $0,75 \%$ e $1,0 \%$ de glicerol não suportaram a mesma tensão aplicada ao filme com $0,5 \%$ de glicerol antes de se romperem, apresentaram-se menos rígidos e mais flexíveis. As diferentes concentrações utilizadas do plastificante não interferiram nos parâmetros de elongação e teste de perfuração.

\section{AGRADECIMENTO}

Â Escola de Agronomia da Universidade Federal de Goiás pela oportunidade e disponibilidade de laboratórios e materiais para o desenvolvimento deste trabalho.

Todos os autores declararam não haver qualquer potencial conflito de interesses referente a este artigo. 


\section{REFERÊNCIAS BIBLIOGRÁFICAS}

AMERICAN SOCIETY FOR TESTING AND MATERIAL. ASTM D 882 - 10: Standard test method for tensile properties of thin plastic sheeting. Philadelphia, 2010.

ASSIS, O. B. G. Alteração do caráter hidrofílico de filmes de quitosana por tratamento de plasma de hmds. Química Nova. v.33, n. 3, p. 603-606, 2010.

ASSIS, O. B. G.; BRITO, D.; FORATO, L. A. O uso de biopolímeros como revestimentos comestíveis protetores para conservação de frutas in natura e minimamente processadas. Boletim de Pesquisa e Desenvolvimento. São Carlos, p. 23, 2009.

BOURTOOM, T.; CHINNAN, M. S. Preparation and properties of rice starche-chitosan blend biodegradable film. Food Science and Technology. Trivandrum, v. 41, n. 4, p. 1633-1641, 2008.

CARVALHO, R. M. Desenvolvimento e caracterização de biofilmes à base de gelatina. Campinas, SP. Dissertação de Mestrado. Universidade de Campinas - UNICAMP, 1997.

DIAS, A. B. Desenvolvimento e caracterização de filmes biodegradáveis obtidos de amido e de farinha de arroz. Florianópolis, SC. Dissertação de Mestrado. Universidade Federal de Santa Catarina - UFSC, 2008.

DIAS, M. V. Filmes a base de quitosana incorporados com $\alpha$-tocoferol e montmorilonita para aplicação em embalagens funcional $\mathrm{e}$ antioxidante. Lavras, MG. Tese de Doutorado. Universidade Federal de Lavras - UFLA, 2013.

FERREIRA, D. F. SISVAR: um programa para análises e ensino de estatística. Revista Symposium (Lavras), v. 6, p. 36-41, 2008

GALDEANO, M. C. Filmes e laminados biodegradáveis de amido de aveia com diferentes plastificantes, produzidos por casting e extrusão. Londrina, PR. Tese de Doutorado. Universidade Estadual de Londrina - UEL, 2007.

GALDEANO, M. C.; MALI, S.; GROSSMANN, M. V. E.; YAMASHITA, F.; GARCIA, M. A. Effects of plasticizers on the properties of oat starch films. Materials Science and Engineering, v. 29, n. 2, p. 532-538, 2009.

GARCIA, A.; SPIM, J. A.; SANTOS, C. A. Ensaio dos Materiais. 2. ed. Rio de Janeiro, RJ, LTC, 260 p. 2000.

LI, Y. et al. Preparation and functional properties of blend films of gliadins and chitosan. Carbohydrate Polymers, Barking, v. 81, n. 2, p. 484-490, 2010.

MALI, S.; GROSSMANN, M. V. E.; YAMASHITA, F. Filmes de amido: produção, propriedades e potencial de utilização. Semina: Ciências Agrárias. Londrina, v. 31, n. 1, p. 137156, 2010.

MALI, S., KARAM, L. B, RAMOS, L. P.; GROSSMANN, M. V. E. Relationships among the composition and physicochemical properties of starches with the characteristics of their films. Journal of Agricultural and Food Chemistry, Washington, v. 52, n. 25, p. 7720-7725, 2004.

OLIVEIRA, L. M. Ensaios para avaliação de embalagens plásticas flexíveis. Campinas: Centro de Tecnologia de Embalagem, CETEA, v. 103, p. 219, 1996.

ROCHA, G. O.; FARIAS, M. G.; CARVALHO, C. W. P.; ASCHERI, J. L. R.; GALDEANO, M. C. Filmes compostos biodegradáveis a base de amido de mandioca e proteína de soja. Polímeros, São Carlos, v. 24, n. 5, p. 587-595, 2014.

RODRÍGUEZ, F. J.; GALOTTO, M. J.; GUARDA, A.; BRUNA, J. E. Modification of cellulose acetate films using nanofillers based organoclays. Journal of Food Engineering, London, v. 110, p. 262-268, 2012.

SEBASTIÃO, V. C. J. Técnicas de Caracterização de Polímeros. São Paulo: Artliber Editora, p. 341-348. 2003.

SILVA, M. G.; SILVA, S. B.; SILVA, J. ; SANTIN, C. K.; SOUZA, D.; FROTA JR, M. Caracterização de filmes à base de quitosana, In: XI Congresso Brasileiro de Engenharia Química em Iniciação Científica. Campinas, Anais. v. 1, p. 1710-1715, 2015.

SOBRAL, P. J. A. Influência da espessura de biofilmes feitos à base de proteínas miofibrilares sobre suas propriedades funcionais. Pesquisa Agropecuária Brasileira, Brasília, v. 35, n. 6, p. 
1251-1259, 2000.

SZYMANSKA, E; WINNICKA, K. Stability of chitosan - a challenge for pharmaceutical and biomedical applications. Marine Drugs, v. 13, p. 1819-1846, 2015.

VIÉGAS, L. P. Preparação e caracterização de filmes biodegradáveis a partir de amido com quitosana para aplicação em embalagens de alimentos. Campos dos Goytacazes, RJ, Dissertação de Mestrado. Universidade Estadual do Norte Fluminense Darcy Ribeiro - UENF, 2016. 